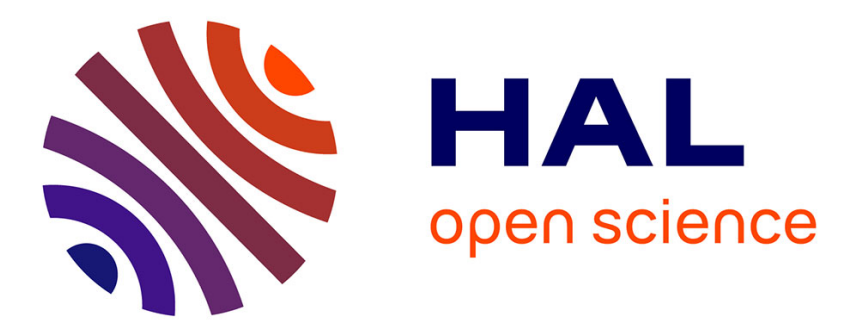

\title{
Thermoelectric and micro-Raman measurements of carrier density and mobility in heavily Si-doped GaN wires
}

Pierre Tchoulfian, Fabrice Donatini, François Levy, Benoît Amstatt, Amélie Dussaigne, Pierre Ferret, Etienne Bustarret, Julien Pernot

\section{To cite this version:}

Pierre Tchoulfian, Fabrice Donatini, François Levy, Benoît Amstatt, Amélie Dussaigne, et al.. Thermoelectric and micro-Raman measurements of carrier density and mobility in heavily Si-doped GaN wires. Applied Physics Letters, 2013, 103 (20), pp.202101. 10.1063/1.4829857 . hal-00905599

\section{HAL Id: hal-00905599 \\ https://hal.science/hal-00905599}

Submitted on 18 Nov 2013

HAL is a multi-disciplinary open access archive for the deposit and dissemination of scientific research documents, whether they are published or not. The documents may come from teaching and research institutions in France or abroad, or from public or private research centers.
L'archive ouverte pluridisciplinaire HAL, est destinée au dépôt et à la diffusion de documents scientifiques de niveau recherche, publiés ou non, émanant des établissements d'enseignement et de recherche français ou étrangers, des laboratoires publics ou privés. 


\title{
Thermoelectric and micro-Raman measurements of carrier density and mobility in heavily Si-doped GaN wires
}

\author{
P. Tchoulfian, ${ }^{1,2, a)}$ F. Donatini, ${ }^{2}$ F. Levy, ${ }^{1}$ B. Amstatt, ${ }^{1}$ A. Dussaigne, ${ }^{1}$ P. Ferret, ${ }^{1}$ \\ E. Bustarret, ${ }^{2}$ and J. Pernot ${ }^{2,3, a)}$ \\ ${ }^{1}$ CEA-LETI, MINATEC Campus, 17 rue des Martyrs, F-38054 Grenoble Cedex 9, France \\ ${ }^{2}$ Institut Néel, CNRS and Université Joseph Fourier, 25 rue des Martyrs, BP 166, F-38042 Grenoble Cedex 9 , \\ France \\ ${ }^{3}$ Institut Universitaire de France, 103 boulevard Saint-Michel, F-75005 Paris, France
}

(Received 19 July 2013; accepted 28 October 2013; published online 11 November 2013)

\begin{abstract}
Combined thermoelectric-resistivity measurements and micro-Raman experiments have been performed on single heavily Si-doped $\mathrm{GaN}$ wires. In both approaches, similar carrier concentration and mobility were determined taking into account the non-parabolicity of the conduction band. The unique high conductivity of Si-doped $\mathrm{GaN}$ wires is explained by a mobility $\mu=56 \mathrm{~cm}^{2} \cdot \mathrm{V}^{-1} \cdot \mathrm{s}^{-1}$ at a carrier concentration $\mathrm{n}=2.6 \times 10^{20} \mathrm{~cm}^{-3}$. This is attributed to a more efficient dopant incorporation in Si-doped $\mathrm{GaN}$ microwires as compared to Si-doped GaN planar layers. (C) 2013 AIP Publishing LLC. [http://dx.doi.org/10.1063/1.4829857]
\end{abstract}

Wire-based devices are promising candidates towards improved electronics and opto-electronics applications. Due to its direct wide band gap $(\sim 3.4 \mathrm{eV}), \mathrm{GaN}$ is the material of choice for optoelectronics applications. In recent years, GaN-based wires have thus attracted considerable interest because of desirable intrinsic properties such as small footprint, increased active region area in core-shell structures, and improved strain relaxation. ${ }^{1-3}$ Similar to the planar epitaxial case, control of doping is essential to obtain highperformance wire-based devices. Localized large carrier concentrations are, for example, required in wire-based devices to decrease series resistances and to improve current injection schemes into the wires. The knowledge of the electrical properties (carrier concentration and mobility) in wire-based semiconductor devices is therefore crucial and is obtained primarily by measuring wires at the single-wire level.

Even in this configuration, access to the mobility and carrier concentration as generally measured by Hall-effect measurements in thin films remains a challenge in wires. Although Hall-effect measurement has been recently reported in nanowires, ${ }^{4-6}$ the measurement and its analysis might be demanding in highly-doped $\mathrm{GaN}$ wires since the measured Hall voltage is inversely proportional to the carrier concentration and depend on the contact geometry on the microwire. Field-effect transistor provides another helpful configuration in order to evaluate the carrier concentration in single low-doped wires. The highly doped material and the microscale of our Si-doped GaN wires have prevented its use. Furthermore, the accuracy of this approach relies on the difficult estimate of both the gate capacitance and interface charges.

In a previous work, ${ }^{7}$ we reported the very low resistivity of single heavily $\mathrm{Si}$-doped GaN wires but a complementary characterization technique was found to be necessary in order to explain quantitatively this particular behavior. In this Letter, we report on the measurement of carrier concentration and

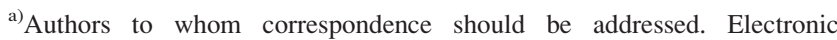
addresses: pierre.tchoulfian@cea.fr and julien.pernot@grenoble.cnrs.fr
}

mobility in single Si-doped GaN microwires grown by catalyst-free metal-organic vapour phase epitaxy (MOVPE). These highly conductive Si-doped GaN wires were studied by means of thermoelectrical measurements and compared to local optical experiments. First, combined resistivitythermoelectric measurements were used to determine both the carrier concentration and the mobility. Second, confocal micro-Raman experiments on non-intentionally doped (NID) and Si-doped GaN wires were compared. From this comparison, the lower branch of the coupled phonon-plasmon of the Si-doped GaN wires was simulated in order to obtain locally both the carrier concentration and the microscopic mobility. The agreement between the results from the two techniques is highlighted. Finally, the unique high conductivity of Si-doped $\mathrm{GaN}$ wires is discussed.

$\mathrm{Si}$-doped wurtzite $\mathrm{GaN}$ wires with radius $\mathrm{r}$ around $1 \mu \mathrm{m}$ (defined as hexagon side length) and length $1=20 \mu \mathrm{m}$ were grown using catalyst-free MOVPE $\mathrm{M}^{3,8,9}$ on N-polar GaN freestanding substrates. Wires were grown along [0001] $(\mathrm{N} \text {-polarity) })^{8}$ at $1000{ }^{\circ} \mathrm{C}$ using trimethylgallium and ammonia precursors, while silane was used as a low-cost precursor to achieve intentional n-type doping. Combined thermoelectric-resistivity measurements were performed on single Si-doped GaN wires. Resistivity $\rho$ combines the effect of carrier concentration $\mathrm{n}$ and mobility $\mu$ through the expression $\rho=1 /(\mathrm{e} \times \mathrm{n} \times \mu)$, where $\mathrm{e}$ is the electronic charge. Thermoelectric measurements are well adapted to doped semiconductors as they are based on majority carrier distribution asymmetry at the Fermi level and allow to probe small changes in the scattering rate and the density of states at Fermi level. ${ }^{10}$ For degenerate semiconductors, thermoelectric measurements have proven to be an effective approach to probe directly the carrier concentration in nanowires. ${ }^{11-14}$ In GaN wires, no study has however combined thermoelectric measurements with resistivity measurements in order to determine both the mobility and the carrier concentration.

In order to perform these two measurements on our microscale $\mathrm{Si}$-doped GaN wires, a specific e-beam lithography 


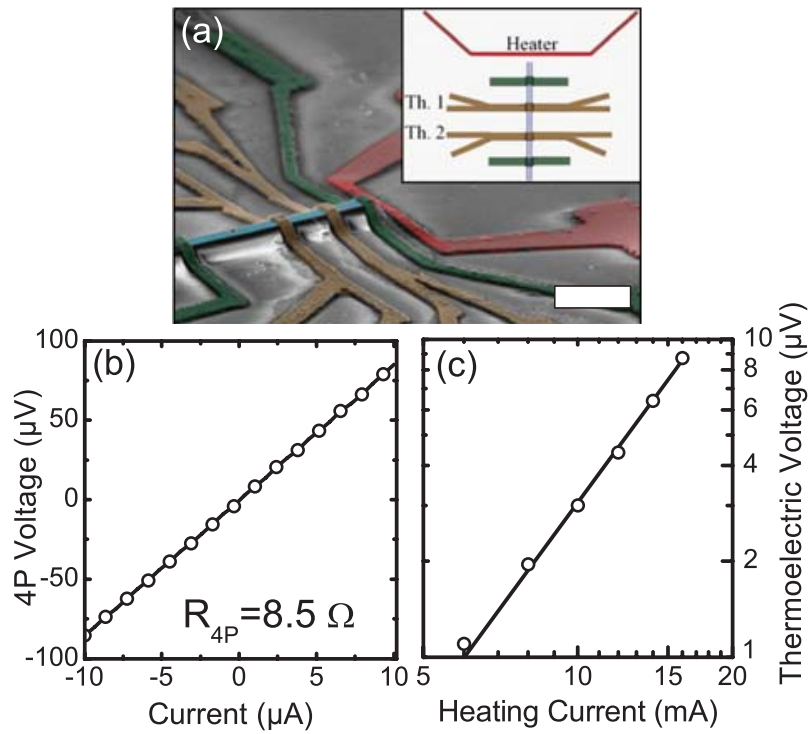

FIG. 1. (a) FESEM image of a connected Si-doped GaN microwire for combined thermoelectric-resistivity measurements. Scale bar: $5 \mu \mathrm{m}$. Inset: Schematic of the contact geometry. (b) Four-probe current-voltage characteristic of the Si-doped GaN wire \#2. (c) Thermoelectric voltage in the Sidoped $\mathrm{GaN}$ wire \#1 as a function of the heater current.

setup has been used to connect single microwires using electron beam lithography assisted with CL mapping. ${ }^{15}$ First, wires were mechanically dispersed on a $500 \mathrm{~nm}$ (thermally grown) $\mathrm{SiO}_{2} / \mathrm{Si}$ wafer and buried under a $2 \mu \mathrm{m}$-thick photoresist. Blind exposure and subsequent partial development allowed uncovering the top facet of every wire. The obtained quasi-flat surface facilitates e-beam lithography. Then, an e-beam resist was spun onto the wafer, and wires were located under this resist using their CL signal and eventually exposed according to the lithography pattern. Finally, contact electrodes were obtained by evaporation of $\mathrm{Ti} / \mathrm{Al}$ $(30 \mathrm{~nm} / 240 \mathrm{~nm})$ and subsequent lift-off.

Figure 1(a) shows a field-emission scanning electron microscope (FESEM) picture of a wire after completion of the process steps for thermoelectric-resistivity measurements. In the four-probe configuration, a current $\mathrm{I}_{\mathrm{s}}$ is sourced through the outer metallic electrodes located on the wire, while the voltage drop $\mathrm{V}_{4 \mathrm{P}}$ between the inner electrodes is measured. The resistivity $\rho$ is then calculated using the equation: $\rho=\left(V_{4 \mathrm{P}} \times \mathrm{A}\right) /\left(I_{\mathrm{S}} \times L\right)$, where $\mathrm{L}$ is the distance between the two inner electrodes and $\mathrm{A}=3 \sqrt{3} / 2 \times(r)^{2}$. A typical IV curve obtained in four-probe configuration is plotted in Figure 1(b) and the measured resistivity values for the two investigated Si-doped wires are reported in Table I.

Figure 1(a) also depicts the configuration to perform thermoelectric measurements in the same region as for resistivity measurements, with a microfabricated heater and two

TABLE I. Resistivity $\rho(\mathrm{m} \Omega \cdot \mathrm{cm})$, Seebeck coefficient $\mathrm{S}\left(\mu \mathrm{V} \cdot \mathrm{K}^{-1}\right)$, carrier concentration $\mathrm{n}\left(\mathrm{cm}^{-3}\right)$, and mobility $\mu\left(\mathrm{cm}^{2} \cdot \mathrm{V}^{-1} \cdot \mathrm{s}^{-1}\right)$ for two Si-doped GaN wires.

\begin{tabular}{lccccc}
\hline \hline Wire & Doping & $\rho$ & $\mathrm{S}$ & $\mathrm{n}$ & $\mu$ \\
\hline$\# 1$ & $\mathrm{Si}$ & 0.43 & $-22 \mp 1$ & $2.6 \times 10^{20}$ & 56 \\
$\# 2$ & $\mathrm{Si}$ & 1.5 & $-58 \mp 3$ & $6 \times 10^{19}$ & 69 \\
\hline
\end{tabular}

sets of thermometers (Th. 1 and 2) which simultaneously serve as electrodes for electrical measurements. The sourcing of a current $I_{h}$ in the heater creates a thermal gradient $\Delta T$ along the structure with isothermal lines parallel to the heater line. $\Delta \mathrm{T}$ was quantified by probing the change in four-probe resistivity of the "thermometer" metallic lines which have been calibrated using a well-controlled heater system. The generated thermoelectric voltage $\mathrm{V}_{\text {th }}$ across the two thermometers was measured as reported in Figure 1(c). Joule heating from the heater is responsible for the generated $V_{\text {th }}$ as shown by the almost quadratic variation of $V_{\text {th }}$ versus $I_{h}$ (power coefficient is 2.1-2.6). Neglecting the Seebeck coefficient of the metal contacts, the Seebeck coefficient $\mathrm{S}$ for GaN simply writes $S=-\frac{V_{t h}}{\Delta T}$.

As shown in Figure 2(a), $V_{\text {th }}$ scales linearly as a function of $\Delta \mathrm{T}$ whose value is tuned by the heating electric current. Seebeck coefficients equal to $-58 \mp 3 \mu \mathrm{V} / \mathrm{K}$ (wire \#2) and $-22 \mp 1 \mu \mathrm{V} / \mathrm{K}$ (wire \#1) are found for the two studied wires. The negative sign of the Seebeck coefficient indicates that electron diffusion dominates and demonstrates that Si-doped $\mathrm{GaN}$ wires are effectively $n$-type semiconductors. In the framework of Boltzmann's transport theory, the semiclassical Mott relation can be used to evaluate the carrier concentration in degenerate semiconductors. ${ }^{10}$ A derivation taking into account an energy-dependent relaxation time and the non-parabolicity of the conduction band was described as ${ }^{16}$

$$
\begin{aligned}
S= & -\frac{8 \times \pi \times\left(\frac{\pi}{3}\right)^{5 / 3} \times k_{b}{ }^{2} \times T}{|e| \times h^{2}} \times \frac{1}{n^{2 / 3}} \\
& \times\left[\mathrm{m}^{*} \times(\mathrm{s}+1-\lambda)\right]_{\mathrm{E}=\mathrm{Ef}},
\end{aligned}
$$

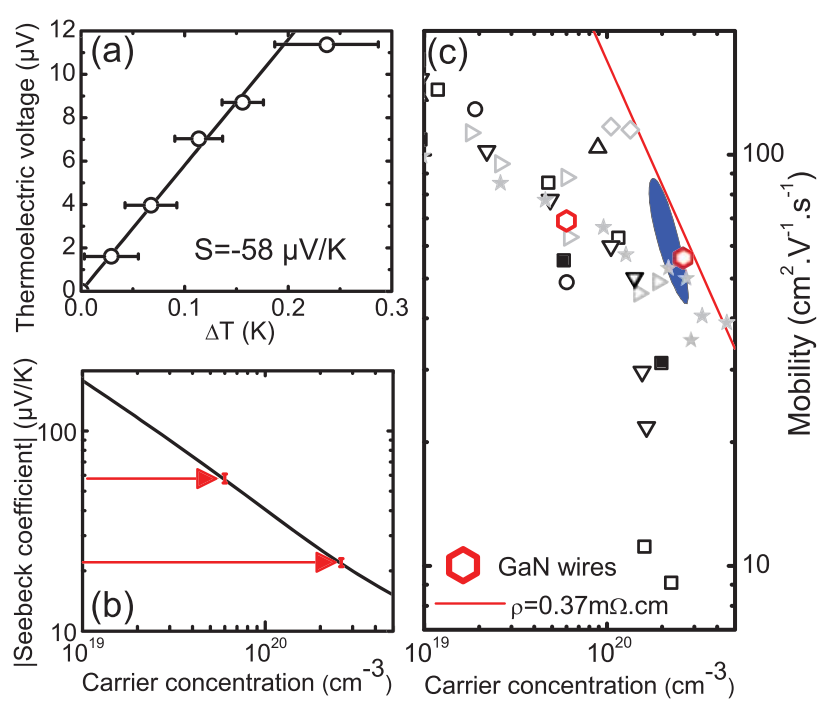

FIG. 2. (a) Thermoelectric voltage versus generated temperature difference $\Delta \mathrm{T}$ in the Si-doped GaN wire \#2 showing a linear behavior. (b) Seebeck coefficient versus carrier concentration in $\mathrm{n}-\mathrm{GaN}$ for two Si-doped wires. (c) Mobility as a function of carrier concentration in n-doped GaN. Experimental mobilities are reported for $\mathrm{n}-\mathrm{GaN}$ films grown by MOVPE (empty symbols), and MBE (full symbols) with either silicon doping ${ }^{22-26}$ (black symbols) or germanium doping $^{25-27}$ (gray symbols) and for two Si-doped GaN wires (red symbols). Isoresistivity line for the experimental value of the most conductive Si-doped $\mathrm{GaN}$ microwire from Ref. 7 is reported. Results from micro-Raman experiments are illustrated by the blue area. 
with $\mathrm{s}$ being the scattering parameter associated with the dominant scattering mechanism and $\lambda=3 \times \frac{\mathrm{n}}{\mathrm{m}^{*}} \times \frac{\mathrm{dm}^{*}}{\mathrm{dn}}$ being the effect of the non parabolicity of the conduction band. ${ }^{16}$ The non-parabolicity is included by using the momentum effective mass ${ }^{17} m^{*}(E)=m_{0}{ }^{*}\left[1+2 M_{1} E+3 M_{2} E^{2}+4 M_{3} E^{3}\right]$ with $\mathrm{m}_{0}{ }^{*}$ $=0.183 \mathrm{~m}_{0} \quad\left(\mathrm{~m}_{0}\right.$ as the electron mass), $\mathrm{M}_{1}=0.177, \mathrm{M}_{2}=$ -0.0169 , and $\mathrm{M}_{3}=0.460$. Ionized impurity scattering is known to be the main scattering mechanism for heavily doped GaN material. ${ }^{18}$ In order to calculate the value for the scattering parameter s, Brooks-Herring theory ${ }^{19}$ for ionized impurity scattering was employed. The effect of the non-parabolicity and the multi-ion screening correction ${ }^{20}$ were included in the model. ${ }^{21}$

Figure 2(b) shows the calculated Seebeck coefficient as a function of the carrier concentration. It can be inferred that $\mathrm{n}=2.6 \times 10^{20} \mathrm{~cm}^{-3}$ for wire \#1 and $\mathrm{n}=6 \times 10^{19} \mathrm{~cm}^{-3}$ for wire \#2 corresponding, respectively, to $\mu=56 \mathrm{~cm}^{2} \cdot \mathrm{V}^{-1} \cdot \mathrm{s}^{-1}$ and $\mu=69 \mathrm{~cm}^{2} \cdot \mathrm{V}^{-1} \cdot \mathrm{s}^{-1}$. This analysis can be compared to Ref. 11 where the authors assume energy-independent scattering and a constant effective mass. As an example, Seebeck coefficient $\mathrm{S}=35 \mu \mathrm{V} / \mathrm{K}$ leads in our analysis to $\mathrm{n}=1.26 \times 10^{20} \mathrm{~cm}^{-3}$ as compared to $\mathrm{n}=7 \times 10^{19} \mathrm{~cm}^{-3}$ in their work. Given the microscale of the studied wires, one can compare the mobility dependence on carrier concentration for GaN films with the one measured in $\mathrm{GaN}$ wires. Experimental data for $\mathrm{n}-\mathrm{GaN}$ films ${ }^{22-27}$ with different growth methods (MOVPE and MBE) and different dopant atoms ( $\mathrm{Si}$ and $\mathrm{Ge}$ ) are thus reported in Figure 2(c), and their comparison with $\mathrm{GaN}$ wires will be discussed later.

Combined thermoelectric-resistivity measurements allow probing the carrier concentration and mobility in the $\mathrm{Si}$-doped $\mathrm{GaN}$ wires, but this approach requires timeconsuming technological work on a single wire. In order to confirm the measured electrical properties, local microRaman experiments have been performed on single standing Si-doped and NID GaN wires. Confocal micro-Raman with a spatial resolution around $1 \mu \mathrm{m}^{3}$ has long been used in planar $\mathrm{n}$-doped GaN material in order to study $\operatorname{strain}^{28,29}$ and doping properties (mainly carrier concentration). ${ }^{29-33}$

For micro-Raman measurements, a microscope equipped with a $\times 100$ objective (numerical aperture NA 0.9) was used both to focus the laser light at $514.5 \mathrm{~nm}$ (power below $1 \mathrm{~mW}$ ) on the sample and to collect the backscattered light into a Jobin-Yvon 64000 spectrometer equipped with a nitrogen-cooled detector. According to group theory for the wurtzite structure, the predicted zone-center optical modes are $\mathrm{A}_{1}(\mathrm{z})+2 \mathrm{~B}_{1}+\mathrm{E}_{1}(\mathrm{x}, \mathrm{y})+2 \mathrm{E}_{2}$. The $\mathrm{A}_{1}, \mathrm{E}_{1}$, and the two $\mathrm{E}_{2}$ are Raman active, while the two $B_{1}$ modes are optically silent. Additionally, the polar nature of $\mathrm{A}_{1}$ and $\mathrm{E}_{1}$ modes result in the splitting of these modes into longitudinal optical (LO) and transverse optical (TO) components: $\mathrm{A}_{1}(\mathrm{LO}, \mathrm{TO})$ and $\mathrm{E}_{1}(\mathrm{LO}, \mathrm{TO})$. In the classical Porto notation, the scattering configuration was $z(x,-) \bar{z}$ ( $\mathrm{z}$ is parallel to the c-axis). Contrary to the transport measurement, the results were obtained on as grown "vertical" wires, not on "horizontal" wires dispersed onto a foreign substrate. According to the Raman selections rules in this configuration, only the two $E_{2}$ (high and low) and the $\mathrm{A}_{1}(\mathrm{LO})$ modes should be observed. They were analyzed in order to evaluate the structural quality and the carrier concentration and mobility in the GaN wires.
Figure 3(a) shows typical Raman spectra for standing NID and Si-doped GaN wires. First, selection rules are not respected in any of the two samples, as illustrated by the presence of $\mathrm{E}_{1}(\mathrm{TO})$ at $558 \mathrm{~cm}^{-1}$. The particular wire tip shape might alleviate the selection rules by allowing internal reflections. In both wires, $\mathrm{E}_{2}$ (high) is clearly visible and peaks at $567 \mathrm{~cm}^{-1}$. The position of $\mathrm{E}_{2}$ (high) is that of its relaxed bulk value $^{29}$ indicating relaxed GaN material in wires even for high silicon-doping. The width of the $\mathrm{E}_{2}$ (high) peak is $4.2 \mathrm{~cm}^{-1}$ for the Si-doped wires and $3.8 \mathrm{~cm}^{-1}$ for the NID wires. It is well established that in doped polar semiconductors, when the plasmon frequency approaches the LO phonon frequency, their macroscopic electric fields strongly interact. This leads to the formation of two coupled LO phonon-plasmon modes (LPPs), the lower branch labeled LPP- and the higher LPP + . The simulation of these peaks provides a direct insight into the carrier concentration and the mobility of the material. The LPP + is observed at $739 \mathrm{~cm}^{-1}$ in the case of the NID GaN in Figure 3(a). In a low-doped material such as the NID wires, the phonon-plasmon coupling manifests itself only in the $\mathrm{LPP}+$ and thus the peak observed at $533 \mathrm{~cm}^{-1}$ can be attributed to $\mathrm{A}_{1}$ (TO).

In the NID wires, the observed $\mathrm{E}_{1}(\mathrm{TO})-\mathrm{A}_{1}(\mathrm{TO})$ splitting is $25 \mathrm{~cm}^{-1}$ which is close to the reported bulk value of 25 to $29 \mathrm{~cm}^{-1} \cdot{ }^{28,31}$ Therefore, the intermixing between the $A_{1}$ and $E_{1}$ modes in the wires is small and cannot explain the violation of Raman selection rules in the backscattering geometry for the wires. It can be concluded that the observed LPP + mode is due to LO phonons of $\mathrm{A}_{1}$ symmetry. The LPP + peak position $\left(739 \mathrm{~cm}^{-1}\right)$ was attributed to regions with low carrier concentration around 2-3 $\times 10^{17} \mathrm{~cm}^{-3}$ using a reported correlation. ${ }^{32}$

In the highly conductive $\mathrm{Si}$-doped $\mathrm{GaN}$ wires, no LPP+ peak could be resolved in the spectral range of $735-4000 \mathrm{~cm}^{-1}$, probably because of a large plasmon damping. For a

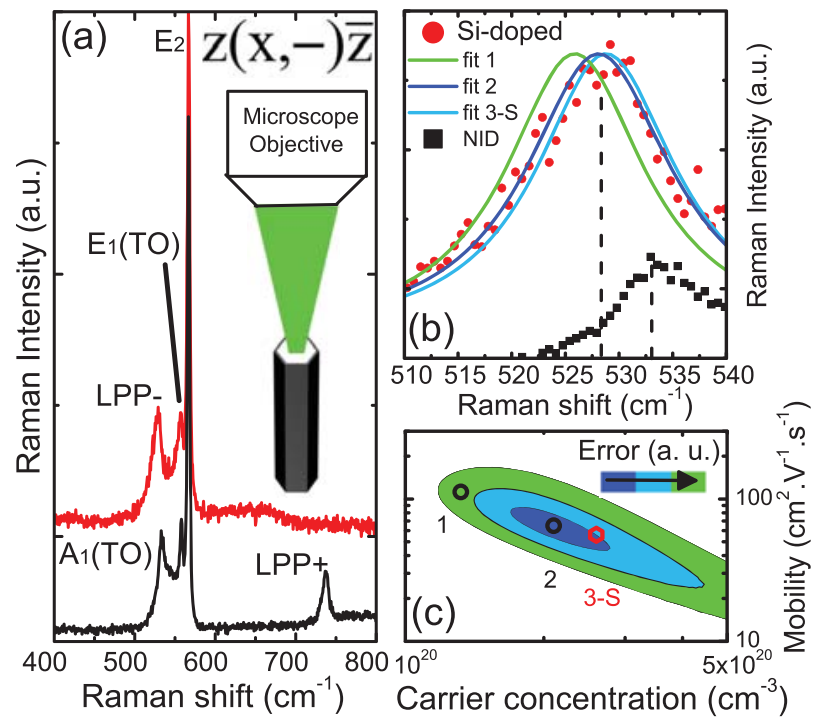

FIG. 3. (a) Raman spectra for a NID GaN wire (black line) and a Si-doped $\mathrm{GaN}$ wire (red line). Inset shows a schematic view of the micro-Raman experiment. (b) Raman spectra showing three simulations of the LPP- corresponding to three sets of the free parameters (carrier concentration and mobility). Set \#3 corresponds to parameters measured for wire \#1. (c) Colorcoded map of the deviation between simulation and experiment with open symbols corresponding to fits in (b). 
thorough evaluation of both the carrier concentration $\mathrm{n}$ and the mobility $\mu$ in the $\mathrm{Si}$-doped wires, a line-shape simulation of the LPP - was used where both the contribution of the deformation potential and the electro-optical mechanisms were taken into account. ${ }^{34}$ The Raman scattering efficiency is then described as $I(w) \propto\left[1, C, C^{2}\right] \times \operatorname{Im}\left(\frac{1}{\varepsilon(\omega)}\right)$ and $\varepsilon(\omega)=\varepsilon_{\infty}$ $\times\left[1+\frac{\omega_{L O}^{2}-\omega_{T O}^{2}}{\omega_{T O}^{2}-\omega^{2}-i \gamma \omega}-\frac{\omega_{P}^{2}}{\omega^{2}+i \gamma_{P} \omega}\right]$, where $\varepsilon(\omega)$ is the dielectric function, $\omega$ is the Raman shift, $\varepsilon_{\infty}=5.35$ is the value of high frequency dielectric constant of $\mathrm{GaN}^{35} \mathrm{C}=-5.2$ is the Faust-Henry coefficient for hexagonal $\mathrm{GaN}^{36,37}$ $\omega_{\mathrm{LO}}=735 \mathrm{~cm}^{-1}$ is the $\mathrm{A}_{1}(\mathrm{LO})$ frequency, ${ }^{28} \gamma=6 \mathrm{~cm}^{-1}$ is the phonon damping constant ${ }^{33}$ and $\omega_{\mathrm{TO}}=533 \mathrm{~cm}^{-1}$ is the $\mathrm{A}_{1}$ (TO) frequency as obtained from the NID wire spectrum. The plasma frequency $\omega_{\mathrm{p}}$ and plasmon damping constant $\gamma_{P}$ are linked to the electrical properties (carrier concentration and mobility) by $\omega_{\mathrm{P}}=\sqrt{\frac{4 \pi \mathrm{e}^{2} \times \mathrm{n}}{\mathrm{m}^{*}\left(\mathrm{E}_{\mathrm{f}}\right) \times \varepsilon_{\infty}}}$ and $\gamma_{P}=\frac{\mathrm{e}}{\mathrm{m}^{*}\left(\mathrm{E}_{\mathrm{f}}\right) \times \mu \times \mathrm{c}}$ with $m *\left(E_{f}\right)$ as the effective mass taking into account the non parabolicity of the conduction band at the Fermi level. The carrier concentration and the mobility are thus the only free parameters of the simulation. In order to illustrate this approach, Figure 3(b) shows three simulations (fits 1-3) corresponding to the three sets of free parameters (carrier concentration and mobility) defined in Figure 3(c). Fit \#3 corresponds to parameters obtained from the combined thermoelectric-resistivity measurements on wire \#1 while fit \#2 reflects the best agreement with the Raman experiment. The deviation between the model and the experiment was calculated using a mean squared error method over a wide range of carrier concentrations and mobilities and the result is illustrated in Figure 3(c) as an error color map. It provides a two-dimensional mobility-carrier concentration space to estimate the most likely combination of carrier concentration and mobility. The best fit of the LPP - was obtained using a carrier concentration and a mobility around $\mathrm{n}=2.1 \times 10^{20} \mathrm{~cm}^{-3}$ and $\mu=65 \mathrm{~cm}^{2} \cdot \mathrm{V}^{-1} \cdot \mathrm{s}^{-1}$. The uncertainty around these values can be quantified by defining a best-fit region in Figure 3(c). The best-fit region of the micro-Raman study is shown in Figure 2(c) to range from $\mathrm{n}=1.77 \times 10^{20} \mathrm{~cm}^{-3}\left(\mu=77 \mathrm{~cm}^{2} \cdot \mathrm{V}^{-1} \cdot \mathrm{s}^{-1}\right)$ to $\mathrm{n}=2.7 \times 10^{20} \mathrm{~cm}^{-3}\left(\mu=44 \mathrm{~cm}^{2} \cdot \mathrm{V}^{-1} \cdot \mathrm{s}^{-1}\right)$. This agrees well with values inferred from combined thermoelectric-resistivity measurements for wire \#1. Although less precise than the thermoelectric approach, this indicates that micro-Raman spectroscopy provides a non-contact and non-destructive probe for carrier concentration and mobility determination in standing wires based on polar material.

The mobility measured for the Si-doped wire \#1 and a wire resistivity $(\rho=0.37 \mathrm{~m} \Omega \cdot \mathrm{cm})$ from our previous work ${ }^{7}$ are reported in Figure 2(c) as red symbols and lines and can be compared to literature on $\mathrm{n}-\mathrm{GaN}$ films where data for $\mathrm{Ge}$ doped $\mathrm{GaN}$ are reported with gray symbols and data for Si-doped films with black symbols. Since isoresistivity lines are parallel to the red line showing $\rho=0.37 \mathrm{~m} \Omega \cdot \mathrm{cm}$, Figure 2(c) highlights the superiority of Ge doping with respect to Si doping in planar epitaxial layers. This was explained by a structural degradation in Si-doped GaN films where tensile strain induced cracks appear from carrier concentration $\mathrm{n}=1-2 \times 10^{19} \mathrm{~cm}^{-3}$. These cracks hampered the electrical measurements and the use of conventional Ga-polar
Si-doped films with carrier concentration larger than around $10^{20} \mathrm{~cm}^{-3} \cdot{ }^{25}$ The wire geometry and the N-polarity might help to explain why the measurement is possible in these wires, as opposed to planar layers of same doping level. ${ }^{38}$ The unique conductivity in microwire \#1 was explained by a combination of a mobility $\mu=56 \mathrm{~cm}^{2} \cdot \mathrm{V}^{-1} \cdot \mathrm{s}^{-1}$ with a large carrier concentration $\mathrm{n}=2.6 \times 10^{20} \mathrm{~cm}^{-3}$. The closest reported measurement in $\mathrm{GaN}: \mathrm{Si}$ layers has a carrier density $\mathrm{n}=2.4 \times 10^{20} \mathrm{~cm}^{-3}$ with a decreased mobility $\mu=9 \mathrm{~cm}^{2} \cdot \mathrm{V}^{-1} \cdot \mathrm{s}^{-1}$ ascribed to self-compensation phenomenon. $^{22}$ However, the impact of cracks on the measured carrier density and mobility is not known in the latter sample. Our measurements show that dopant incorporation is more efficient at this high carrier concentration in Si-doped wire-based devices as compared to conventional epitaxial Si-doped layers. The improved mobility could be explained by a lower compensation in the wires or by the presence of some additional scattering mechanisms in planar layers with cracks. The comparison between electrical measurements and $\mathrm{Si}$ atoms density measurements would help to clarify the compensation in the wires. Conductivity values for $\mathrm{Si}$-doped $\mathrm{GaN}$ wires are comparable to the conductivity of Ge-doped layers. The mobility in these GaN microwires is not decreased by strong surface-related effects, as often reported on highly-doped GaN nanowires. ${ }^{39}$

To summarize, carrier concentration and mobility have been evaluated by two approaches in the same region of Sidoped GaN microwires grown by catalyst-free MOVPE. In a first approach, combined thermoelectric-resistivity measurements were carried out while in a second approach, local micro-Raman experiments were performed. Both approaches were found to give similar results with a carrier concentration $\mathrm{n}=2.6 \times 10^{20} \mathrm{~cm}^{-3}$ and a mobility $\mu=56 \mathrm{~cm}^{2} \cdot \mathrm{V}^{-1} \cdot \mathrm{s}^{-1}$. A more efficient dopant incorporation in $\mathrm{Si}$-doped $\mathrm{GaN}$ wires as compared to Si-doped films is shown to be responsible for the high conductivity, with values comparable to Ge-doped films. Such a high conductivity demonstrates the technological potential of microwires and paves the way to more efficient current injection schemes and improved series resistances in wire-based devices.

Research was partially supported by the French National Research Agency within the FIDEL Project (No. ANR-11NANO-27).

The authors acknowledge G. Feuillet and D. Tainoff for fruitful discussions and the Nanofab team at Institut Néel for the use of their facilities and their technical assistance.

${ }^{1}$ Y. Huang, X. Duan, Y. Cui, and C. M. Lieber, Nano Lett. 2, 101 (2002).

${ }^{2}$ A. L. Bavencove, D. Salomon, M. Lafossas, B. Martin, A. Dussaigne, F. Levy, B. Andre, P. Ferret, C. Durand, J. Eymery, L. S. Dang, and P. Gilet, Electron. Lett. 47, 765 (2011).

${ }^{3}$ R. Koester, J. S. Hwang, D. Salomon, X. Chen, C. Bougerol, J.-P. Barnes, Le Si Dang, L. Rigutti, A. de Luna Bugallo, G. Jacopin, M. Tchernycheva, C. Durand, and J. Eymery, Nano Lett. 11, 4839-4845 (2011).

${ }^{4}$ J. P. Degrave, D. Liang, and S. Jin, Nano Lett. 13, 2704 (2013).

${ }^{5}$ K. Storm, F. Halvardsson, M. Heurlin, D. Lindgren, A. Gustafsson, P. M. Wu, B. Monemar, and L. Samuelson, Nat. Nanotechnol. 7, 718-722 (2012). ${ }^{6}$ C. Blömers, T. Grap, M. I. Lepsa, J. Moers, S. Trellenkamp, D. Grützmacher, H. Lüth, and T. Schäpers, Appl. Phys. Lett. 101, 152106 (2012).

${ }^{7}$ P. Tchoulfian, F. Donatini, F. Levy, B. Amstatt, P. Ferret, and J. Pernot, Appl. Phys. Lett. 102, 122116 (2013). 
${ }^{8}$ X. J. Chen, G. Perillat-Merceroz, D. Sam-Giao, C. Durand, and J. Eymery, Appl. Phys. Lett. 97, 151909 (2010).

${ }^{9}$ R. Koester, J. S. Hwang, C. Durand, L. S. Dang, and J. Eymery, Nanotechnology 21, 015602 (2010).

${ }^{10}$ D. C. K. MacDonald, Thermoelectricity: An Introduction to the Principles (Courier Dover Publications, 2006).

${ }^{11}$ C. Lee, G. C. Yi, Y. M. Zuev, and P. Kim, Appl. Phys. Lett. 94, 022106 (2009).

${ }^{12}$ P. M. Wu, W. Paschoal, S. Kumar, C. Borschel, C. Ronning, C. M. Canali, L. Samuelson, H. Pettersson, and H. Linke, J. Nanotechnol. 2012, 1.

${ }^{13}$ Y. M. Zuev, J. S. Lee, C. Galloy, H. Park, and P. Kim, Nano Lett. 10, 3037 (2010).

${ }^{14}$ J. Moon, J. H. Kim, Z. C. Y. Chen, J. Xiang, and R. Chen, Nano Lett. 13, 1196 (2013).

${ }^{15}$ F. Donatini and L. S. Dang, Nanotechnology 21, 375303 (2010).

${ }^{16}$ D. L. Young, T. J. Coutts, V. I. Kaydanov, A. S. Gilmore, and W. P. Mulligan, J. Vac. Sci. Technol. A 18, 2978 (2000).

${ }^{17}$ S. Shokhovets, O. Ambacher, B. Meyer, and G. Gobsch, Phys. Rev. B 78 035207 (2008).

${ }^{18} \mathrm{H}$. Morkoç, Handbook of Nitride Semiconductors \& Devices: Electronic and Optical Processes in Nitrides (Wiley, 2008), Vol. 2.

${ }^{19}$ D. C. Look, Electrical Characterization of GaAs Materials and Devices (Wiley, New York, USA, 1989), pp. 77-78.

${ }^{20}$ J. Meyer and F. Bartoli, Phys. Rev. B 36, 5989 (1987).

${ }^{21}$ Screening by free carriers was found to reduce the scattering parameters $\mathrm{s}$ from 2 to around 1.4-1.5 for carrier concentration ranging from $10^{19}$ to $5 \times 10^{20} \mathrm{~cm}^{-3}$. The correction due to multi-ion screening was accounted and it resulted in adding around 0.1 to the value considering only single ion screening as in original Brooks-Herring theory.

${ }^{22}$ I. Halidou, Z. Benzarti, Z. Chine, T. Boufaden, and B. El Jani, Microelectron. J. 32, 137 (2001).

${ }^{23}$ X. Liu, L. Wang, D. C. Lu, D. Wang, X. Wang, and L. Lin, J. Cryst. Growth 189/190, 287 (1998).
${ }^{24}$ H. M. Ng, D. Doppalapudi, T. D. Moustakas, N. G. Weimann, and L. F. Eastman, Appl. Phys. Lett. 73, 821 (1998).

${ }^{25}$ S. Fritze, A. Dadgar, H. Witte, M. Bügler, A. Rohrbeck, J. Bläsing, A. Hoffmann, and A. Krost, Appl. Phys. Lett. 100, 122104 (2012).

${ }^{26}$ M. Wieneke, H. Witte, K. Lange, M. Feneberg, A. Dadgar, J. Bläsing, R. Goldhahn, and A. Krost, Appl. Phys. Lett. 103, 012103 (2013).

${ }^{27}$ P. R. Hageman, W. J. Schaff, J. Janinski, and Z. Liliental-Weber, J. Cryst. Growth 267, 123 (2004)

${ }^{28}$ U. Haboeck, H. Siegle, A. Hoffmann, and C. Thomsen, Phys. Status Solidi C 0, 1710 (2003).

${ }^{29}$ S. Tripathy, S. J. Chua, P. Chen, and Z. L. Miao, J. Appl. Phys. 92, 3503 (2002).

${ }^{30}$ E. Frayssinet, W. Knap, S. Krukowski, P. Perlin, P. Wisniewski, T. Suski, I. Grzegory, and S. Porowski, J. Cryst. Growth 230, 442 (2001).

${ }^{31}$ T. Kozawa, T. Kachi, H. Kano, Y. Taga, M. Hashimoto, N. Koide, and K. Manabe, J. Appl. Phys. 75, 1098 (1994).

${ }^{32}$ C. Wetzel, W. Walukiewicz, E. Haller, J. Ager, I. Grzegory, S. Porowski, and T. Suski, Phys. Rev. B 53, 1322 (1996).

${ }^{33}$ F. Demangeot, J. Frandon, M. A. Renucci, C. Meny, O. Briot, and R. L. Aulombard, J. Appl. Phys. 82, 1305 (1997).

${ }^{34}$ H. Harima, S. Nakashima, and T. Uemura, J. Appl. Phys. 78, 1996, (1995).

${ }^{35}$ A. S. Barker and M. Ilegems, Phys. Rev. B 7, 743 (1973).

${ }^{36}$ C. Wetzel, W. Walukiewicz, and J. W. Ager III, Electron - Phonon Scattering in Si Doped GaN, in III-V Nitrides, edited by F. Ponce, T. D. Moustakas, I. Akasaki, and B. Monemar (Mater. Res. Soc. Symp. Proc., 1997), Vol. 449, pp. 567.

${ }^{37}$ Value of coefficient $\mathrm{C}$ has been found to have a very limited influence on the line shape and frequency of the coupled mode.

${ }^{38}$ P. Prystawko, M. Leszczynski, B. Beaumont, P. Gibart, E. Frayssinet, W. Knap, P. Wisniewski, M. Bockowski, T. Suski, and S. Porowski, Phys. Status Solidi B 210, 437 (1998).

${ }^{39}$ A. Motayed, M. Vaudin, A. V. Davydov, J. Melngailis, M. He, and S. N. Mohammad, Appl. Phys. Lett. 90, 43104 (2007). 